AC 2012-5601: BEST ZONE PAPER: IMPLEMENTING SOCIAL LEARNING STRATEGIES: TEAM TESTING

Prof. Rebecca A. Bates, Minnesota State University, Mankato 

TESTING

Rebecca A Bates, Minnesota State University, Mankato

Andrew Petersen, University of Toronto Mississauga 


\title{
Implementing Social Learning Strategies: Team Testing
}

\begin{abstract}
This paper describes how to provide collaborative learning opportunities and fast feedback on exam performance by adding a team component to examinations. The method is supported by research in collaborative and active learning pedagogy and has been applied to computer science courses ranging from first-year programming to graduate-level artificial intelligence. This paper relates the use of team tests in two different university settings, with a range of implementations. Furthermore, it offers suggestions for customizing the technique to fit a specific classroom environment.
\end{abstract}

\section{INTRODUCTION}

Finding the time and opportunity to incorporate active and collaborative learning in your classes can be challenging. Team testing is a collaborative learning activity with low implementation costs and multiple advantages for both students and faculty. Along with the obvious benefit of developing team problem solving and discussion skills, students receive fast feedback on their performance, the instructor spends less time reviewing the exam (in class and with individuals), and the classroom environment benefits from the added value placed on collaboration and reciprocal learning. This paper describes a number of variations on the team testing idea and discusses how factors in the course affect the type of team test to develop.

In a team test, the students complete an individual test paper as well as a group test paper. The individual component enforces individual accountability and allows the instructor to ask questions in formats that do not naturally benefit from a group discussion. The group component asks the students to evaluate others' ideas and to synthesize a solution that incorporates the best ideas generated by the members of the group. Due to the need to compare and defend ideas, questions on the group test naturally elicit and evaluate higher-level cognitive functions like analysis, evaluation and transfer. ${ }^{1}$ By doing so, team testing converts the evaluation environment into a learning environment. Depending on instructor goals, team tests can be structured to reinforce evaluation goals or learning environment goals.

Conventional wisdom holds that test periods are lost instructional time, but we recall that in our own experience as students, good exams often led to a more complete understanding of the material by forging relationships between domains and encouraging deeper insights. One student who participated in a team test reported, "You can collaborate and discuss what the general consensus of the answer is. ... You [can] see why their answer might be more correct." By leveraging the motivation that an examination conveys, team testing encourages active discussions between well-prepared individuals and fosters an ideal collaborative learning environment.

In addition to evaluating and encouraging higher levels of proficiency, team testing reduces the latency between evaluation and feedback. Exams give faculty an important opportunity to evaluate students, but they also serve to inform students of their progress and to point out areas 
where work is needed. Feedback should be timely to reduce exposure to content misperceptions and reinforcement of correct understandings, ${ }^{2,3}$ but the time required to provide feedback to students is dependent on faculty time and the availability of grading support. The pressures on faculty time are discussed at length at conferences and meetings, and institutional budgets dictate whether grading support is available. In practice, this means that feedback is provided more slowly than desired, but team testing provides immediate feedback by exposing students to their peers' understanding of the material and testing their ability to contribute to the group's solution. By providing students with more timely feedback, team testing reinforces foundational knowledge that the remainder of the course requires.

In the next section, we describe the baseline team testing implementation. Section 3 provides the pedagogical theory supporting the idea, and Section 4 presents our experiences using variants on the baseline team testing implementation in the classroom.

\section{IMPLEMENTING TEAM TESTING}

\subsection{Giving the Exam}

The exam format depends on the ultimate goal of the group exam: evaluating content knowledge in a group context or creating a learning environment. To assess individual ability, an exam is given to each student. The individual exam is evaluated and typically forms the majority component of the student's score. After the individual exam, there is a group component, which can either be a required part of the exam (evaluation goal) or considered a bonus (learning environment goal). Groups of 3 to 4 students collaborate on the group test. Larger groups reduce the impact of individual voices, and groups of two often suffer from a dominant (but not necessarily stronger) partner. The group exam can be given in the same class period, if time is available, or in the following period. Providing a gap between the two exams can be beneficial because students can shore up weaknesses in their understanding brought to light by the individual exam. However, not too much time should be allowed, since more misconceptions in understanding will be uncovered by the group exam than by the individual exam, and it is important to provide fast feedback.

Before the exam, students must prepare sufficiently to be ready to actively engage during the exam. Without adequate preparation, students may find it too easy to passively rely on the group consciousness and may not be capable of identifying gaps in their own knowledge during the discussion. To encourage individual accountability and active discussion during the exam, the students must follow two rules.

1. Each student must write some of the answers.

2. All students must agree on every answer submitted.

When there is a "hung jury", students can be encouraged to record the top two positions with a supporting argument for each. This becomes an answer the group can agree on.

During the group exam, the instructor's job is to facilitate discussion. As with any type of classroom problem solving, the instructor should move from group to group to keep students on task, to refocus or raise questions when necessary. If the primary goal is to facilitate further learning rather than evaluate current knowledge, instructors can provide hints or feedback about 
trains of thought. At the same time, the instructor can encourage individual accountability by drawing out students not actively participating or by keeping time and calling for groups to select a new student to record answers.

\subsection{Setting the Questions}

The individual component and group component should test the same domain of material so that the students gain the benefit of fast feedback. The questions on the two components may be the same or may differ in the level of proficiency exercised and evaluated. The type of questions featured on a group component will differ based on the level of the students in the class and the instructor's learning objectives. The instructor may generate new questions for the group exam, but in many cases, asking the students to compare, analyze, or evaluate their answers from the individual exam enables a deeper exploration of the question in the collaborative context.

In early classes, where goals are often related to content knowledge and application within a defined setting and where students are less experienced learners, the questions may focus on factual or procedural knowledge and might not differ from the individual exam. Presenting the same problem on both exams prepares the students for discussion and encourages them to have a stake in a specific answer. Students at this level benefit from comparing answers and debating which is most correct. ${ }^{1}$

For more advanced courses with mature learners, questions that focus on factual knowledge are less useful on the group exam as they do not foster in-depth discussion. The format favors openended problems that require students to evaluate and compare several possible solutions to reach a solution or that ask them to evaluate the context in which their knowledge is being applied.

\section{SUPPORTING IDEAS}

\subsection{Theoretical Foundations}

The theoretical foundation for this teaching method stems first from the work of Vygotsky who described a social constructivist framework for learning. In this type of setting, learning is enhanced by interaction with others beyond that which is possible individually. ${ }^{4,5}$ Building community via discussion and collaborative learning can support the integration of academic and social experiences upon which student success has been shown to depend. ${ }^{6,7}$

The ideas of active, collaborative, and reciprocal learning stem from Vygotsky's framework. In general, active and collaborative learning promote greater positive attitudes towards learning and the subject matter. ${ }^{8}$ In reciprocal learning, students take turns teaching others. This is built into the structure of this approach, which strengthens student understanding of the material for both strong and weak students. Guided instruction, ${ }^{9}$ where scaffolding built by the teacher is reduced until the student has responsibility for learning, also motivates this approach: exams are used as a source of learning and content discussion, with students building community through their interaction with each other. Along the way, they build communication skills and communication abilities. In terms of team building, this approach has a low time-cost. By using the two simple rules presented in Section 2.1 to guide the process, we address the finding that students succeed 
best in teamwork when there are specific instructions about listening, leadership, consensus building and conflict resolution. ${ }^{10,11}$

Even though collaborative learning has been supported by the National Science Foundation and recommended by the education community, it is still not widely practiced in engineering classrooms. ${ }^{12}$ When implementing active learning, faculty sometimes find it difficult to give up their role as controllers of knowledge. ${ }^{13}$ However, giving an exam is an expected part of both traditional and active classrooms. As such, team testing may be a relatively comfortable step to take towards a more active classroom. Within computer science, team programming has been shown to enhance learning and community (e.g., McDowell et al. ${ }^{14}$ ). Team testing can augment a class using team programming or provide similar benefits when used alone.

Feedback is generally considered important in learning and there is a growing body of work (and technology ${ }^{*}$ ) related to providing fast feedback (e.g., Chen et al. ${ }^{2}$ and Mehta ${ }^{3}$ ). Unfortunately, not much, if anything, has been published on the use of discussion as a fast feedback mechanism or the return speed of graded exams and assignments. However, structured discussion with peers is a well-known method for increasing engagement and enhancing learning. ${ }^{15,16}$ Psychology research has shown that reinforcement, through punishment and/or rewards, is important to learning and that learning is easier when the reinforcement closely follows the action. ${ }^{17}$ Team testing provides the reward of rapid feedback in two ways. First, it provides students with solution feedback and secondly, it provides feedback about their own performance and how it relates to the performance of their peers.

\subsection{Justification}

Cooperative learning in a testing environment is beneficial because students develop group interaction and individual reasoning skills with the encouragement of a reward. The students have spent time considering the problems before discussion, which encourages them to become more involved. In fact, team-testing discussions are amongst the most lively and energetic in our courses, in part because of emotional reactions to learning answers but also because each student is prepared for the discussion. The students are clearly connecting the content to a social learning environment, and as a result, they are better able to recall and reason about course material. ${ }^{18}$

Many teachers spend a significant amount of class time going over the correct responses to an exam, or worry that they cannot take the time to do this without losing content coverage. Rather than spend the same amount of time going over the exam, a team test allows students to discover, justify and own the answers. By having students spend time working through the exam with each other, the students become teachers, participating in reciprocal learning.

For planning teacher-to-classroom feedback, the group exams give a better picture of which concepts were globally missed or were difficult. As with any exam, this can be useful feedback

\footnotetext{
* The Web-based Interactive Science and Engineering Learning Tool at Oregon State University is an example of fast-feedback technology. (Koretsky, M. Web-based Interactive Science and Engineering Learning Tool. https://secure.engr.oregonstate.edu/che/WISE/. )
} 
about the teaching of the content, with even more weight because the results have been filtered through both individual minds and group consideration. When the teacher discusses the exam, the concepts missed after the group exam can be the focus, thus reducing faculty review time.

\subsection{Benefits}

One model of active learning separates "doing" from "observing" and differentiates between dialog "with self" and "with others". ${ }^{8}$ Group tests contain room for both types of dialog. First, the solo test forces to the student to hold a dialog with self, with the instructor as audience. Next, the group section asks the students to enter into a dialog with others -- potentially with problems they have already considered and prepared answers for. While this dialog enhances learning, it also enhances the sense of community and has been shown to improve student attitudes toward the discipline and course. ${ }^{19}$ Rather than prolonging a competitive evaluation atmosphere, a collaborative environment is available for students who prefer collaboration over competition.

In terms of feedback, after taking a team test, especially one identical to the individual exam, students leave the room knowing which of their answers are likely to be correct. One problem in test-taking is when smart students apply their own logic to problem solving but do not necessarily have the grounding knowledge. The logic may be solid but the answer is not correct. Discussion and skeptical questioning leads the group closer to a correct answer. As one student reported, "It was pretty productive because you could give an answer ... but someone could give a rebuttal. ... It would help you out in your understanding."

Comparing performance on team and individual tests has made it possible to give students feedback not only on their content knowledge but also on their confidence in their knowledge. One student reported, "Having someone else's word that something is correct feels like you are given more confidence in what is right and wrong." Another was the only one of his group to get the answer correct on the individual test but was unable to persuade his peers to change their minds. After written and verbal encouragement, this student began to advocate his knowledge more strongly. At other times, we have observed students who are prepared but not confident in their knowledge be pleasantly surprised to find that they are at the same level, if not higher, than their peers.

\section{TEAM TESTING IN THE CLASSROOM}

Team testing has been employed in a total of nine different courses at two separate institutions: Minnesota State University, Mankato (MSU) and the University of Toronto Mississauga (UTM). The classes spanned the curriculum, ranging from first year programming to third year operating systems to graduate level natural language processing, so they display a variety of sizes (5-50 students), learners (novice to independent), and content (logical and algorithmic problem solving as well as design of large systems).

In this section, we introduce factors that affect the effectiveness of team testing and influence how it should be deployed. We report anecdotal evidence from our own experiences with team testing, relating students concerns and feedback wherever possible. The discussion focuses on the conditions in the classes that caused us to alter the team testing implementation. 


\subsection{Class Size}

The authors have successfully used team testing in classes of up to 50 students. However, colleagues have successfully employed various collaborative testing techniques in courses with more than 500 students. The primary consideration is physical space, which should be plentiful enough to allow groups to interact comfortably. Because discussion can get lively and faculty interaction is helpful, we have found these techniques to work especially well in classes from 2030. On the other end of the spectrum, we have employed team testing in classes with as few as 56 students. In this case, a single discussion group works, even though there are more than the preferred 3-4 students, because in very small classes, a group dynamic of discussion and problem solving often exists already.

\subsection{Reward Systems}

The most important variable that affects how students perceive team testing is its impact on their final grade. In its original form, we imagined team testing to be an opportunity to practice good discussions and to convert an evaluation environment into a learning environment. Students in a learning environment should feel comfortable practicing new skills, which suggests that the evaluation weight should be low or nonexistent. At the same time, team tests work because the students come to the activity prepared and motivated, so we cannot simply eliminate the "test" component of team tests.

The original versions of team testing at MSU made the team test voluntary and offered extra credit for participating. In our experience, offering just $5 \%$ bonus credit on the individual exam causes an overwhelming fraction of students to remain for the team-test. The bonus points offer a tangible reward to the students, countering the stress that comes with exams and grades, while providing motivation to invest energy and preparation in a good outcome. As a result, students did not appear to feel stressed by the addition of the group component of the exam, but they did actively engage in the exercise.

In contrast, the academic culture at UTM made it difficult to offer bonus credit on an exam, so the group component of the exam was weighted as a small fraction $(10 \%)$ of the overall exam mark. As a result, initial student reaction to the team test was quite different at UTM, with students expressing concern about being placed in a group with perceived "weaker" members and feeling stress preparing for the group activity. Instead of viewing the team test as an opportunity to collaborate, the students reacted negatively to a personal evaluation being affected by a factor outside of their control.

The instructors also perceived noticeable differences between the two exam environments. By offering extra credit for the team test, the MSU instructor was able to treat the exercise as a collaborative learning activity. She circulated among the groups, asked questions, and redirected groups that strayed off track. The UTM instructor, however, felt uncomfortable intervening in individual groups and felt that the exercise was an evaluation. 
Nevertheless, in both cases, discussion within the groups was vigorous, and after the experience, students were pleased with the group exam. Both sets of students viewed the group discussion as a means for improving their overall mark, and many students enjoyed the activity.

\subsection{Marking}

The reward system chosen also impacts how the team test is marked. When assessing collaborative learning, the basic approach is to provide independent and interdependent assessment for group work. ${ }^{8}$ The independent component of the exam maintains individual responsibility. If the reward is minimal and the focus is on learning, rather than evaluation, then the grades for group exams can be coarse, reducing grading time and ignoring small differences between groups. If the focus is on evaluation, then more care needs to be spent on the marking to provide formative feedback on the group's effectiveness.

In our experience, due to the low weight of the group component and the focus on learning rather than evaluation, grading on group exams can be generous, not requiring a 100\% correct answer to get full marks, making the reward positive for the students while still allowing for fast feedback. One student in particular was concerned that the other members of the group would not share his desire for excellence: "I really have a hard time doing the group thing as I strive to get the best grade and working in a group seldom allows for that opportunity." A small reward provides motivation, and keeping the reward small enough to avoid further stress about the results of the group exam reduces complaints and classroom tension if a student feels he or she has been placed in a "bad" group.

\subsection{Exam Composition}

Team testing supports many different kinds of questions, and the relationship between questions on the individual exam varies depending on the instructor's objectives. At both MSU and UTM, the instructors favored reusing questions from the independent component of the test. In our experience, relating the questions on the two exam components increases discussion since each student has spent some time thinking about the question and formulating an answer to which they have some emotional attachment. MSU had some success reusing exactly the same questions, and they found that solutions to group exam questions were noticeably stronger than typical answers on the individual exam. UTM tended to ask follow-on questions and saw similar results. Re-using questions also relieves one concern students reported about team tests - that the material on the group component would be significantly more difficult.

Regardless of the type of questions used, the group component should contain far less material than the individual component. When using a reduced set of problems rather than a full test, we found that students would delve deeply into related issues, starting discussions about theory and context that are rare in a regular classroom. In an electric circuit theory class, team testing was used on weekly quizzes. A short, two-problem quiz was given and then the same two problems were given to student pairs. After the first week, the team quiz was reduced to one problem, because students spent so much time discussing the application of theory. As a result, they were often able to show better understanding during this phase than on their individual quiz. 


\subsection{Instructor Time}

Team testing does create some additional work for the teacher: creating a second exam and grading additional questions. This can be minimized by using the same (or at least related) questions on the group component and by coarsely grading the team work. Furthermore, marking time on the individual component is reduced since less feedback needs to be provided. Since the students have discussed the questions, they often better understand how they earned their grade, so students ask fewer questions about marking in office hours and by email. Instead, some student groups used office hours to continue the group discussion with instructor adjudication.

\subsection{Exam Timing}

When giving the team tests, the class structure made a difference in the method and the time allowed for team tests. When the class session is long enough (1.5 to 2 hours), immediately following the individual exam with the group exam works well. When there are recitation sections or labs, a portion of that time can be used for the team test instead of using extra lecture time. Giving a team test in the next class period, especially with time at the end for classroom discussion and questions also works, but it weakens the feedback link.

Students who have become accustomed to both the feedback and reward of group exams have asked for them at the final exam. In these cases, a slightly reduced exam time, e.g., from 120 minutes to 90 minutes, allows time for a team test as well as comprehensive coverage of course content. This gives students feedback they might not otherwise receive at the end of a term.

We have not yet tried giving a group exam before the individual exam. We feel this would increase exam preparation time since the group exam questions and individual exam questions would need to be more decoupled for a fair individual assessment. We are also concerned that discussion would be weaker, since the students would enter the group component with less preparation.

\subsection{Group Formation}

For any type of team work, the selection of the teams and the roles assigned to the team members has an impact on the team's success. The goal of group formation is to create effective groups, giving students a fair chance to do well while minimizing the problems that come with group work. We have used a wide variety of heuristics including student physical proximity (both close and distant), splitting up friends/lab partners/long term groups, and coupling weaker and stronger students. With one notable exception, the groups functioned well regardless of their method of creation. In that case, a student acknowledged as being exceptionally strong inadvertently dominated discussion, since his group was unprepared to challenge his ideas. Teacher intervention is required in this situation. The other students may be encouraged to enter the discussion by asking them to justify why they agree with the proposed solution.

At UTM, where the group component is a fraction of the test mark, some students requested that they be informed of group pairings before the exam to facilitate the formation of study groups. 
Despite some initial concern that releasing the pairings would result in the responsibility for studying material being split up among members of the group, we found that the individual exam led students to study all of the material. As a side benefit, some of the study groups that were formed because of the team test continued to meet later in the term.

We have not investigated assigning teams on a long-term basis but believe it would be an interesting avenue for future study. This approach would reinforce the social learning aspect and allow for more flexibility in negotiation of roles within a team.

\section{SUMMARY}

Team testing enhances student learning by providing faster feedback and using collaborative learning to develop higher order cognitive skills. It leverages student desire to thoroughly prepare for evaluation to increase engagement in a focused small-group discussion. In doing so, team testing converts an evaluation environment into a learning environment.

Team testing is a flexible idea which we have successfully implemented in many types of courses for a broad range of students, learning goals, and content foci. Since the exercise often repurposes material already generated for the individual component of the exam and decreases time spent reviewing exam solutions, we have found team testing to be a low-cost method for introducing collaborative learning into the classroom. More importantly, students have responded extremely positively - even going so far as to request a team test for the final exam!

\section{ACKNOWLEDGMENTS}

Thanks to our students who were willing- and often enthusiastic! - participants in this process.

\section{REFERENCES}

\footnotetext{
${ }^{1}$ Biggs, J.B. \& Collis, K.F. 1982. Evaluating the Quality of Learning: The SOLO Taxonomy. New York Academic Press.

${ }^{2}$ Chen, J.C., Whittinghill, D.C., and Kadlowec, J.A. 2006. Using rapid feedback to enhance student learning and satisfaction. Frontiers in Education.

${ }^{3}$ Mehta, S.I. 1995. A Method for Instant Assessment and Active Learning. Journal of Engineering Education, 84: 295.

${ }^{4}$ Vygotsky, L. 1978. Mind and society: The Development of Higher Mental Processes. Cambridge, MA: Harvard University Press.

${ }^{5}$ Vygotsky, L. 1986. Thought and Language, revised American Edition, translated and edited by Alex Kozulin, Cambridge, MA: MIT Press.

${ }^{6}$ Treisman, U. 1992. Studying Students Studying Calculus: A Look at the Lives of Minority Mathematics Students in College. College Mathematics Journal 23(5), pp. 362-372.
} 


\footnotetext{
${ }^{7}$ Wilson, D. 2008. Does it Matter? Psychological Sense of Community \& Belonging in Engineering Education. Frontiers in Education 2008.

${ }^{8}$ Johnson, D.W., Johnson, D.T. \& Smith, K.A. 1998. Active Learning: Cooperation in the College Classroom, $2^{\text {nd }}$ Ed. Edina, MN: Interaction Book Company.
}

${ }^{9}$ Hausfather, S. J. 1996. Vygotsky and schooling: Creating a social context for learning. Action in Teacher Education. (18) 1-10.

${ }^{10}$ Jones, D.W. 1996. Empowering teams in the classroom can work. Journal for Quality and Participation, 19(1), 80-86.

${ }^{11}$ Schmidt, L., Schmidt, J., Colbeck, C., Bigio, D., Smith, P. \& Harper, L. 2003. Engineering students and training in teamwork: How effective? Proc. American Society for Engineering Education Conference.

${ }^{12}$ Smith, K.L., Sheppard, S.D., Johnson, D.W., \& Johnson, R.T. 2005. Pedagogies of Engagement: Classroom Based Practices, Journal of Engineering Education, 94 (1) 87-101.

${ }^{13}$ Bruner, J. 1985. Vygotsky's theory and the activity-oriented approach in psychology. In Culture, Communication, and Cognition: Vygotskian Perspectives, J. V. Wertsch, ed. New York: Cambridge University Press.

${ }^{14}$ McDowell, C., Werner, L., Bullock, H., Fernald, J. 2002. The effects of pair-programming on performance in an introductory programming course. ACM Technical Symposium on Computer Science Education (SIGCSE '02).

${ }^{15}$ Schellens, T. \& Valcke, M. 2004. Collaborative learning in asynchronous discussion groups: What about the impact on cognitive processing? Computers in Human Behavior, 21(6), 957-975.

${ }^{16}$ Wells, G. (Ed.) 2000. Action, Talk, and Text: Learning and Teaching through Inquiry. New York: Teachers College Press.

${ }^{17}$ Ferster, C.B. \& Skinner, B.F. 1957. Schedules of Reinforcement. New York: Appleton-Century-Crofts.

${ }^{18}$ Kapitanoff, S.H. 2009. Collaborative testing: cognitive and interpersonal processes related to enhanced test performance, Active Learning in Higher Education, 10(1), 56-70.

${ }^{19}$ Slusser, S.R. and Erickson, R.J. 2006. Group quizzes: an extension of the collaborative learning process, Teaching Sociology, 34(3), 249-262. 